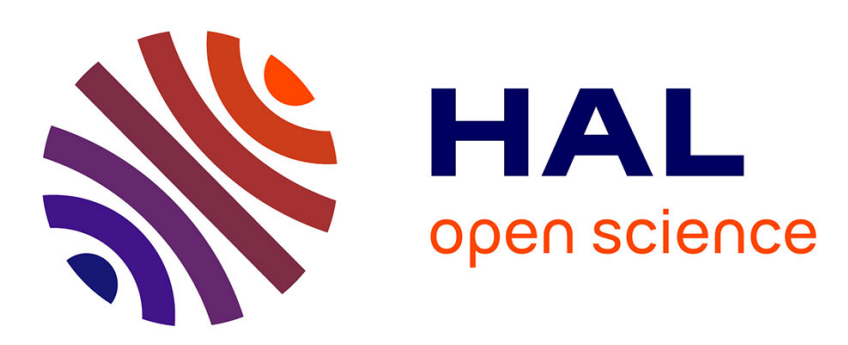

\title{
Evaluating the carbon depletion found by the Stardust mission in Comet $81 \mathrm{P} /$ Wild 2
}

Evgenij Zubko, Karri Muinonen, Yurij Shkuratov, Edith Hadamcik, Anny

Chantal Levasseur-Regourd, Gorden Videen

\section{- To cite this version:}

Evgenij Zubko, Karri Muinonen, Yurij Shkuratov, Edith Hadamcik, Anny Chantal Levasseur-Regourd, et al.. Evaluating the carbon depletion found by the Stardust mission in Comet 81P/Wild 2. Astronomy and Astrophysics - A\&A, 2012, 544, pp.L8. 10.1051/0004-6361/201218981 . hal-00724148

\section{HAL Id: hal-00724148 \\ https://hal.science/hal-00724148}

Submitted on 17 Apr 2016

HAL is a multi-disciplinary open access archive for the deposit and dissemination of scientific research documents, whether they are published or not. The documents may come from teaching and research institutions in France or abroad, or from public or private research centers.
L'archive ouverte pluridisciplinaire $\mathbf{H A L}$, est destinée au dépôt et à la diffusion de documents scientifiques de niveau recherche, publiés ou non, émanant des établissements d'enseignement et de recherche français ou étrangers, des laboratoires publics ou privés. 


\title{
Evaluating the carbon depletion found by the Stardust mission in Comet 81P/Wild 2
}

\author{
E. Zubko ${ }^{1,2}$, K. Muinonen ${ }^{1,3}$, Y. Shkuratov ${ }^{2}$, E. Hadamcik ${ }^{4}$, A.-C. Levasseur-Regourd ${ }^{5}$, and G. Videen ${ }^{6,7}$ \\ ${ }^{1}$ Department of Physics, University of Helsinki, PO Box 64, 00014 Helsinki, Finland \\ e-mail: ezubko@rambler.ru \\ 2 Institute of Astronomy, Kharkov National University, 35 Sumskaya St., 61022 Kharkov, Ukraine \\ 3 Finnish Geodetic Institute, PO Box 15, 02431 Masala, Finland \\ 4 Université Pierre \& Marie Curie, LATMOS, 11 Bd. D’Alembert, 78280 Guyancourt, France \\ 5 Université Pierre \& Marie Curie, LATMOS, BC 102, 4 place Jussieu, 75005 Paris, France \\ 6 Space Science Institute, 4750 Walnut Street, Suite 205, Boulder, CO 80301, USA \\ 7 US Army Research Laboratory, 2800 Powder Mill Road, Adelphi, MD 20783, USA \\ Received 7 February 2012 / Accepted 19 July 2012
}

\section{ABSTRACT}

\begin{abstract}
The low abundance of refractory carbonaceous material in samples collected by Stardust in comet 81P/Wild 2 coma was completely unexpected. If these results are universal to other comets, this necessitates a reformulation of current models of solar system formation. A polarimetric imaging analysis demonstrates that dust is not uniformly distributed within cometary coma, and that the circumnucleus halo region where the dust samples were collected must contain a low population of carbonaceous particles. Such regions are seen in other comets, suggesting that comet 81P/Wild 2 is not unusual and that the anomalous lack of carbon is not necessarily representative of the entire coma.
\end{abstract}

Key words. comets: general - comets: individual: 81 p/Wild 2 - polarization

\section{Introduction}

The low abundance of refractory carbonaceous material in samples collected by Stardust at its closest approach to the comet 81P/Wild 2 nucleus (about $240 \mathrm{~km}$ ) was completely unexpected (e.g., Ishii et al. 2008). Carbon is an essential component of organic material, which is thought to be formed in the interstellar medium by processing a solid mixture of carbon, oxygen, nitrogen, and hydrogen with UV radiation and ion bombardment (Jenniskens et al. 1993; Starukhina \& Shkuratov 1995). Comets presently observed in the solar system are believed to be remnants of planetesimals that contained organic materials and/or amorphous carbon and are expected to preserve pristine material of the interstellar medium. This theory has long been accepted, and indeed, in situ measurements of comet 1P/Halley carried out by the VeGa-1 and 2 spacecraft in 1986 confirmed that all analyzed cometary dust particles contain carbonaceous material, and that in $25 \%$ of the particles, carbon is the dominant component (Kissel et al. 1986; Fomenkova et al. 1992). Similarly, a high proportion of organic matter was found in all 29 particles analyzed with the Cometary and Interstellar Dust Analyzer (CIDA) mass spectrometer during the comet Wild 2 flyby (Kissel et al. 2004), but most of those particles were analyzed at distances greater than $650 \mathrm{~km}$ to the nucleus. Interplanetary dust particles (IDPs) in the Earth's stratosphere (Brownlee et al. 1993; Busemann et al. 2009) also are actively collected. Cometary particles are identified either by their high velocity when entering the Earth's atmosphere (Brownlee et al. 1993) or through dedicated sampling by collecting particles after a close encounter with a comet (Busemann et al. 2009). Dust particles with a cometary origin can also be retrieved from Antarctic snow (Nittler 2010). Cometary dust collected in these ways has a significant carbonaceous component.
The lack of carbonaceous materials in the Stardust samples is unlikely to be caused by interaction of the dust particles with aerogel (e.g., Ishii et al. 2008). Analyzing the aerogel samples, Brownlee et al. (2006) observed that components larger than micron-size were often well preserved, whereas smaller or finer-grained components were strongly modified. We would expect a significant population of super-micron carbonaceous particles that would not have been affected by the aerogel. For instance, Fomenkova et al. (1992) observed that about $25 \%$ of the comet Halley dust particles consist almost entirely of carbonaceous material and some 50\% contain from 10-90\% organic material by mass. A high concentration of carbonaceous materials implies that they not only form the outermost layer of the dust particles but are present throughout the dust particle volume. Therefore, if comet Halley is at all representative, this speaks to a very significant percentage of super-micronsized particles that would not have been affected by the aerogel. Due to the high impact speed $\left(\sim 19 \mathrm{~km} \mathrm{~s}^{-1}\right)$ and relatively long interaction with stratosphere, 5- $\mu \mathrm{m}$ cometary IDPs experience heating to 1100-1200 K (e.g., Brownlee et al. 1993). This significantly exceeds the overall heating of the Stardust samples, which is just several hundreds degree (Brownlee et al. 2000). As a consequence, carbonaceous materials in the Stardust samples should be better preserved compared to the cometary IDPs. Nevertheless, in the former case, their abundance is substantially lower (e.g., Ishii et al. 2008).

The low abundance of refractory carbonaceous materials in 81P/Wild 2 samples is remarkably inconsistent with both the prevalent cometary theory and previous observations. In addition, Stardust revealed various refractory minerals formed in the vicinity of the Sun. As a consequence, it was concluded that $81 P /$ Wild 2 more closely resembles an inner solar system 
asteroid than an outer solar system comet with primitive unaltered dust (Ishii et al. 2008). This conclusion is also consistent with the highly cratered surface morphology found in 81P/Wild 2 (e.g. A'Hearn 2006).

The dissonance between our previous knowledge of comets as reservoirs of pristine material and the findings of the Stardust mission becomes even more pronounced if we take into account the known history of $81 \mathrm{P} /$ Wild 2 . Indeed, this comet is a former Kuiper-belt object whose orbit lay between a perihelion at 4.9 AU and aphelion at $25 \mathrm{AU}$. In 1974, it was diverted into the inner part of the solar system after a close encounter with Jupiter, and currently orbits between 1.6 and 5.2 AU (Ishii et al. 2008). Between 1974 and 2004, the comet experienced only five perihelion passages. Therefore, $81 \mathrm{P} /$ Wild 2 was widely expected to be a fresh comet not significantly exposed to solar radiation.

Stardust findings have had an immediate impact on models of solar system formation. It has been suggested, for instance, that the large-scale radial mixing in the solar nebula had been substantially underestimated (e.g., Ishii et al. 2008; Brownlee et al. 2006; Matzel et al. 2010). Alternative resolutions to the Stardust challenges are that the samples collected from $81 \mathrm{P} /$ Wild 2 are unrepresentative of the entire coma or that this comet is unusual.

\section{Spatial variance of degree of linear polarization}

Polarimetry is the analysis of the polarization state of light scattered by particles, which provides clues to their properties (e.g. morphology, size, composition), especially when complementary information is available (Hadamcik \& Levasseur-Regourd 2003a; Zubko et al. 2009, 2011a). The most common polarization parameter is the degree of linear polarization $P=\left(I_{\perp}-I_{\|}\right) /\left(I_{\perp}+I_{\|}\right)$, where $I_{\perp}$ and $I_{\|}$are the intensities of the scattered electromagnetic fields whose electric fields vibrate perpendicular to and within the scattering plane, respectively (see Fig. 1A). Polarization $P$ is dependent on the phase angle $\alpha$ between the direction of illumination and observation, as seen from the dust.

Polarimetric imaging provides evidence that different types of dust are present in different regions within the cometary coma. The three images in Fig. 1B were obtained at small phase angles for comets C/1995 O1 (Hale-Bopp) (left), 81P/Wild 2 (middle), and 22P/Kopff (right) (Hadamcik \& Levasseur-Regourd 2003b). In all cases polarization varies throughout the coma. The polarization maps of these different comets are remarkably similar, suggesting similarity in the dust properties in these regions. For instance, the innermost coma produces a strong negative polarization of up to $-6 \%$, which is called the circumnucleus halo (Hadamcik \& Levasseur-Regourd 2003b). The material in cometary jets, which is positively polarized, may be superimposed on the image of the halo (e.g. in the C/1995 O1 image of Fig. 1B). The halo is typically a few thousand kilometers across, but the size varies from one comet to another. However, its extent is relatively small, much smaller than the size of the whole coma. When comparing panels in Fig. 1B, one can conclude that the circumnucleus halo of $81 \mathrm{P} /$ Wild 2 is similar to those of other comets. Outside the circumnucleus halo, the polarization is much weaker and tends toward positive values.

Figure 1C shows the phase dependence of the degree of linear polarization of the circumnucleus haloes of different comets adapted from Hadamcik \& Levasseur-Regourd (2003b) for comets 81P/Wild 2, C/1995 O1 (Hale-Bopp), C/1990 K1 (Levy), 47P/Ashbrook-Jackson, and 22P/Kopff. The

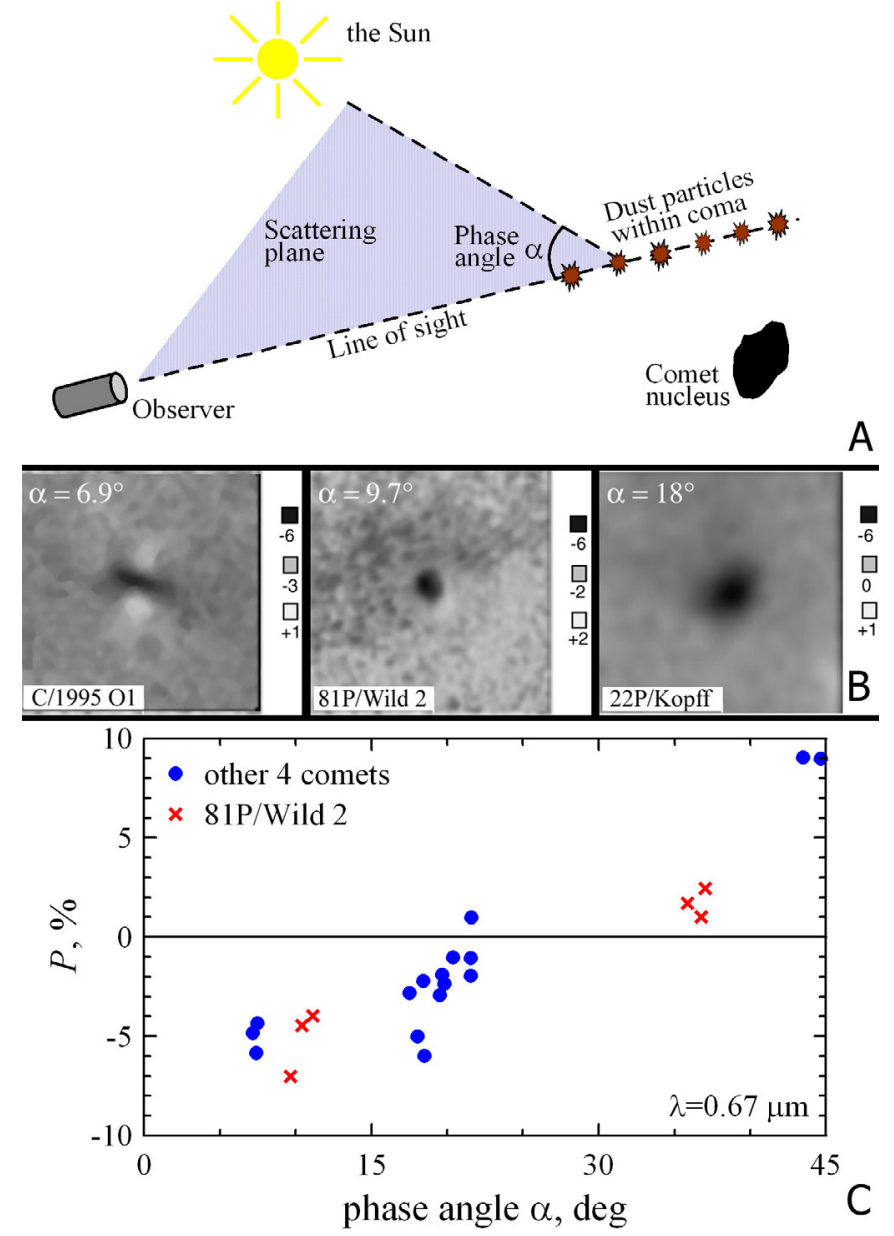

Fig. 1. A) Observation geometry for polarimetric observations of comets. B) Polarimetric images of comets C/1995 O1 (Hale-Bopp) (left), 81P/Wild 2 (middle), and 22P/Kopff (right) obtained at small phase angles. The frame size is equal to $38000 \mathrm{~km}, 9000 \mathrm{~km}$, and $4400 \mathrm{~km}$, respectively. C) Synthetic phase dependence of the degree of linear polarization in the circumnucleus halo.

data points corresponding to $81 \mathrm{P} /$ Wild 2 (red crosses) are consistent with those for other comets (blue points), and all of them form a branch of negative polarization at small phase angles extending to $\alpha \sim 25-30^{\circ}$. This angular profile of the negative polarization is qualitatively similar to that observed in other targets in the solar system (e.g., Johnson et al. 1980; Levasseur-Regourd et al. 1996; Shkuratov et al. 2011; Zellner \& Gradie 1976). An important parameter characterizing the negative polarization branch is the maximum amplitude of the negative polarization. For the circumnucleus haloes, this value $\left|P_{\min }\right| \approx 6 \%$.

\section{Link with chemical composition of dust}

Modeling the light scattered by irregularly shaped particles can provide insight into the properties of particles that have particular polarization features. For highly irregular agglomerates, which would be expected in cometary coma, results show that the scattered light is most strongly dependent on the size of the agglomerates and their complex refractive index. Changes in agglomerate morphology have only a minor effect on the scattering properties, so long as it is highly irregular (Zubko et al. 2008, 2011b; Zubko 2012). The high negative polarization in the circumnucleus halo places a strict material constraint on dust particle absorption (Zubko et al. 2009). We demonstrate this 

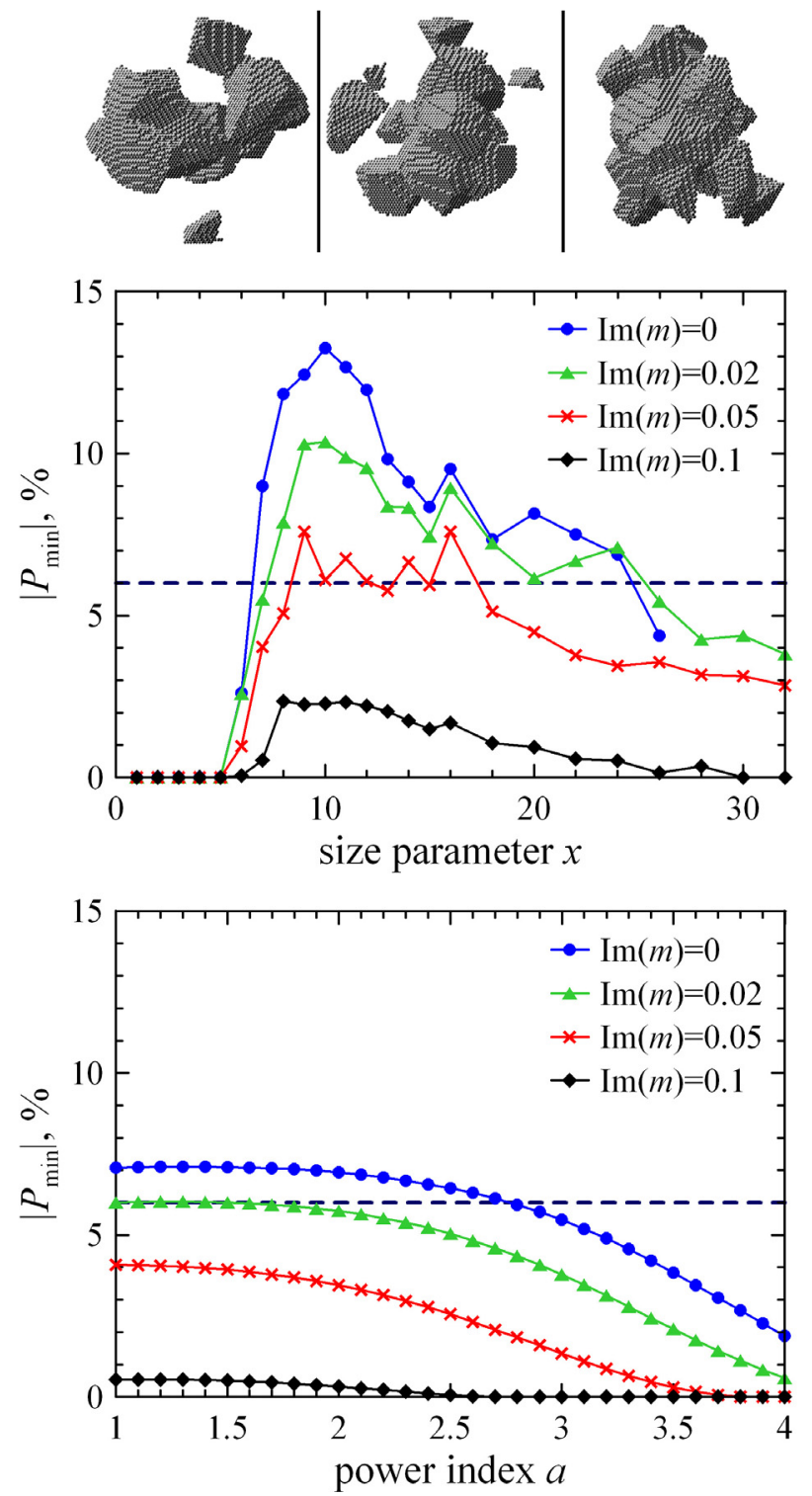

Fig. 2. A) Three samples of agglomerated debris particles. The dependence of the amplitude of minimum polarization $\left|P_{\min }\right|$ on $\left.\mathbf{B}\right)$ the size parameter $x$ and $\mathbf{C}$ ) the power index $a$ of a power-law size distribution for four different refractive indices: $m=1.5+0 \mathrm{i}, m=1.5+0.02 \mathrm{i}$, $m=1.5+0.05 \mathrm{i}$, and $m=1.5+0.1 \mathrm{i}$. The negative polarization of $\left|P_{\min }\right|=6 \%$ measured from the circumnucleus haloes is shown by the dashed line.

feature for highly irregular agglomerated debris particles shown in Fig. 2A by generating model particles that satisfy two widely accepted assumptions about cometary dust morphology, that it be highly irregular and fluffy. These agglomerated debris particles explain the unusual spectral behavior of the negative polarization found in comet 17P/Holmes shortly after its explosion in October 2007 (Zubko et al. 2011a). Significant variations of particle packing density $\rho$ from 0.139 to 0.336 have only a minor impact on the negative polarization (Zubko et al. 2011b), and we consider a packing density $\rho=0.236$ in our calculations. When we consider that the bulk material density for refractory species in comets is in the range $1.5-3.5 \mathrm{~g} / \mathrm{cm}^{3}$, the material density in agglomerated debris particles spans the range $0.35-0.83 \mathrm{~g} / \mathrm{cm}^{3}$, which is consistent with what was determined from the microcraters in the aluminum foil that covered the Stardust sample collector: $0.3-3 \mathrm{~g} / \mathrm{cm}^{3}$ (e.g., Hörz et al. 2006).
The light scattered from agglomerated debris particles is calculated using the discrete dipole approximation (DDA) (e.g., Zubko et al. 2010). Light-scattering properties depend not on particle size but on its ratio to wavelength $\lambda$, quantified by the size parameter $x=2 \pi r / \lambda$, where $r$ is the radius of the circumscribing sphere about the particle. Another important characteristic is the complex refractive index $m$ of the particle material, which relates chemical and mineral composition of the given material with its ability to scatter and absorb light.

Figure $2 \mathrm{~B}$ demonstrates the dependence of $\left|P_{\min }\right|$ on the size parameter $x$ for materials consistent with $\mathrm{Mg}$-rich silicates and organic materials in visible wavelengths (Dorschner et al. 1995; Jenniskens 1993), the two most abundant species within comets (Fomenkova et al. 1992). Mg-rich silicates have little absorption, $\operatorname{Im}(m) \approx 0$. Organic materials have varying absorption depending on the amount of carbon they contain and the processing they have undergone. The shapes of the polarization responses are qualitatively similar in Fig. 2B. The negative polarization does not exist (i.e., $\left|P_{\min }\right|=0$ ) if the particles are smaller than $x \approx 6$. Then, in the range of $x \approx 6-10$, the negative polarization appears and rapidly grows with $x$. An additional increase of $x$ decreases the negative polarization. Material absorption significantly dampens the amplitude of the negative polarization. Indeed, at $\operatorname{Im}(m)=0$, it exceeds $13 \%$, while at $\operatorname{Im}(m)=0.1$, it remains substantially less than $3 \%$ throughout the range of $x$. The negative polarization disappears altogether for sufficiently large particles. Because particles with $\operatorname{Im}(m)=0.1$ cannot produce negative polarization with amplitude greater than $3 \%$, we may immediately conclude that these particles are not the dominant species within the circumnucleus haloes where $\left|P_{\min }\right| \approx 6 \%$. This conclusion holds throughout the range of the real part of refractive index $\operatorname{Re}(m)=1.3-1.7$ (Zubko et al. 2009; Zubko et al. 2011a). Furthermore, it was shown in Zubko et al. (2009) that the negative polarization completely disappears at $\operatorname{Im}(m)=0.2$ and an additional increase of absorption up to $\operatorname{Im}(m)=1.3$ does not produce significant negative polarization.

Real cometary dust is not monodisperse. According to laboratory analyses of micro-craters in the aluminum foil of the Stardust spacecraft, for the range of particle sizes from 0.2 to $10 \mu \mathrm{m}$, dust obeys a power-law size distribution $r^{-a}$, where the power index $a=1.89$ (Price et al. 2010). However, it is important to note that this power index varies for different comets and may also be time-dependent. Figure $2 \mathrm{C}$ demonstrates the dependence of $\left|P_{\min }\right|$ on the power index a over sizes ranging from 0.2 to approximately $6 \mu \mathrm{m}$. The size averaging dramatically suppresses the amplitude of the negative polarization compared to monodisperse particles, since the significant contribution of the small particles does not produce any negative polarization. Since the impact of small particles increases with index $a$, the negative polarization decreases with increasing $a$. As a consequence, the constraint on the material absorption becomes significantly stricter when size averaging is included. Particles of any power-law distribution with $\operatorname{Im}(m)>0.02$ are unable to reproduce the polarimetric properties of circumnucleus haloes. Most carbonaceous materials are highly absorbing. For instance, at $\lambda=0.7 \mu \mathrm{m}$, organic material in a form expected in the diffuse interstellar medium has a refractive index $m=1.566+0.075 \mathrm{i}$ (Jenniskens 1993); whereas amorphous and glassy carbon have $m=2.43+0.59 \mathrm{i}$ and $m=1.8+0.75 \mathrm{i}$, respectively (Duley 1984). It is clear that none of these materials can appear in any of the observed circumnucleus haloes in significant quantities. Thus, the high negative polarizations measured in the circumnucleus halo of $81 \mathrm{P} /$ Wild 2 are consistent with the lack of carbon particles found in the Stardust mission. 
We emphasize that the circumnucleus halo that produces a strong negative polarization is only a small part of the whole coma. The degree of linear polarization in other parts of the coma is much less negative. Moreover, some features, such as cometary jets, do not reveal any negative polarization at all (Hadamcik \& Levasseur-Regourd 2003b). For instance, in the case of comet 81P/Wild 2 presented in Fig. 1B, the negative polarization in the circumnucleus halo is $P=(-6 \pm 1) \%$; whereas a sunward jet shows positive polarization $P=(+1 \pm 0.5) \%$. Simultaneously, the whole coma of 81P/Wild 2 yields an average $P=(-1.8 \pm 0.5) \%$ (Hadamcik \& Levasseur-Regourd 2003b). One can see that the circumnucleus halo does not dominate the total polarization signal. As a consequence, the lack of carbonaceous materials cannot be attributed to the entire coma region, but only to its innermost part. Indeed, the absence of the negative polarization in cometary jets can be explained as having an abundance of highly absorbing materials (see Fig. 2C). However, as was already noticed, strong material absorption is a distinctive feature of various carbonaceous materials.

Our interpretation of the Stardust sampling of the dust particles forming the $81 \mathrm{P} /$ Wild 2 circumnucleus halo slightly contradicts the findings in Sekanina et al. (2004), who attributed the discrete burst activity registered by the Dust Flux Monitor Instrument (DFMI) (e.g., Tuzzolino et al. 2004) to jets emanating from comet $81 \mathrm{P} /$ Wild 2 . Non-jet cometary activity (e.g., Belton 2010) is not considered, but the images analyzed in Sekanina et al. (2004) reveal a substantial level of such activity, which appears as numerous non-collimated fluxes originating from the extended surface areas on $81 \mathrm{P} /$ Wild 2 . It is especially significant that the DFMI sampling of comet 9P/Tempel 1 revealed the same discrete burst activity as in 81P/Wild 2 (e.g., Veverka et al. 2012). However, it is believed that the Stardust trajectory did not cross any specific jet in comet 9P/Tempel 1, suggesting that a significant level of heterogeneity is an inherent property of cometary coma, regardless of the presence of jets. These small-scale spatial inhomogeneities $(\sim 1 \mathrm{~km})$ do not contradict our assumption on the domination in the innermost coma of pre-surface dust particles ejected at low velocity. Other mechanisms can produce coma inhomogeneities, for instance, periodic activity of source(s) of non-accelerated dust particles and/or the impact of radiation pressure (e.g., Fulle 2004). Inhomogeneities in the innermost coma can also result from sublimation of large icy chunks contaminated with refractory cometary species.

\section{Conclusions}

The circumnucleus halo, as detected by polarization, appears to be a ubiquitous, carbon-depleted (or carbon-hidden) feature of comets because it appears in many of the polarization images obtained at small phase angles. Other regions of the coma display significantly lower negative polarizations that are consistent with the presence of absorbing particles. Cometary jets, for instance, have revealed only positive polarization through all phase angles (Hadamcik \& Levasseur-Regourd 2003b), and 81P/Wild 2 displayed such a sunward jet (Hadamcik \& Levasseur-Regourd 2003b). Since high-carbon content has been a characterizing feature in all samplings prior to the Stardust mission, it appears that the anomalous results are due to sampling in this carbon-depleted region. Sampling in this anomalous region may also explain why the crystalline structure of silicates found is different from the chondritic porous interplanetary dust that was expected to be present (Ishii et al. 2008). While the knowledge of the origin and evolution of comets developed before the Stardust mission may not require an immediate revision, the physical mechanisms that produce a circumnucleus halo are currently not well understood. The small size of the halo suggests that it consists of particles that were not accelerated by an expanded gas, like the cometary jets. Its shape may indicate that its dust originated from a relatively large area of the nucleus or that it persists for long periods. Therefore, one can hypothesize that the circumnucleus halo may be caused by near-surface processes directly stimulated by sunlight (e.g., Belton 2010) or through long-term settling of particles within the coma. The preferential selection of non-absorbing particles and the abundance of material resembling chondritic meteorites may provide clues to the halo's origin.

Acknowledgements. This work was partially supported by the Academy of Finland (contract 127461), by the NASA program for Outer Planets Research (grant NNX10AP93G), by the French space agency (CNES) and by the French Programme national de planétologie (PNP). The authors are grateful to the anonymous referee for valuable comments on this paper.

\section{References}

A'Hearn, M. F. 2006, Science, 314, 1708

Belton, M. J. S. 2010, Icarus, 210, 881

Brownlee, D. E., Joswiak, D. J., Love, S. G., et al. 1993, Lunar Planet. Sci., XXIV, 205

Brownlee, D. E., Tsou, P., Aléon, J., et al. 2000, Meteor. Planet. Sci., 35, A35

Brownlee, D., Tsou, P., Clark, B., et al. 2006, Science, 314, 1711

Busemann, H., Nguyen, A. N., Cody, G. D., et al. 2009, Earth Planet. Sci. Lett., 288,44

Dorschner, J., Begemann, B., Henning, T., et al. 1995, A\&A, 300, 503

Duley, W. W. 1984, ApJ, 287, 694

Fomenkova, M. N., Kerridge, J. F., Marti, et al. 1992, Science, 258, 266

Fulle, M. 2004, in Comets II, eds. M. C. Festou, H. U. Keller, \& H. A. Weaver (Tucson: University of Arizona Press), 565

Hadamcik, E., \& Levasseur-Regourd, A.-C. 2003a, A\&A, 403, 7577

Hadamcik, E., \& Levasseur-Regourd, A.-C. 2003b, J. Quant. Spectr. Rad. Trans., 79,661

Hörz, F., Bastien, R., Borg, J., et al. 2006, Science, 314, 1716

Ishii, H. A., Bradley, J. P., Dai, Z. R., et al. 2008, Science, 319, 447

Jenniskens, P. 1993, A\&A, 274, 653

Jenniskens, P., Baratton, G. A., Kouchi, A., et al. 1993, A\&A, 273, 583

Johnson, P. E., Kemp, J. C., King, R., et al. 1980, Nature, 283, 146

Kissel, J., Sagdeev, R. Z., Bertaux, J. L., et al. 1986, Nature, 321, 280

Kissel, J., Krueger, F. R., Silén, J., Clark, B. C., et al. 2004, Science, 304, 1774

Levasseur-Regourd, A. C., Hadamcik, E., \& Renard, J. B. 1996, A\&A, 313, 327

Matzel, J. E. P., Ishii, H. A., Joswiak, D., et al. 2010, Science, 328, 483

Nittler, L. R. 2010, Science, 328, 698

Price, M. C., Kearsley, A. T., Burchell, M. J., et al. 2010, Meteor. Planet. Sci., 45,1409

Sekanina, Z., Brownlee, D. E., Economou, T. E., et al. 2004, Science, 304, 1769 Shkuratov, Y., Kaydash, V., Korokhin, V., et al. 2011, Planet. Space Sci., 59, 1326

Starukhina, L. V., \& Shkuratov, Y. G. 1995, Icarus, 113, 442

Tuzzolino, A. J., Economou, T. E., Clark, B. C., et al. 2004, Science, 304, 1776

Veverka, J., et al. 2012, Icarus, in press

Zellner, B., \& Gradie, J. 1976, AJ, 81, 262

Zubko, E. 2012, in Light Scattering Reviews, ed. A. A. Kokhanovsky (Berlin: Springer-Verlag), 6, 39

Zubko, E., Shkuratov, Y., Mishchenko, M., Videen, G., et al. 2008, J. Quant. Spectr. Rad. Trans., 109, 2195

Zubko, E., Kimura, H., Shkuratov, Y., et al. 2009, J. Quant. Spectr. Rad. Trans., 110,1741

Zubko, E., Petrov, D., Grynko, Y., et al. 2010, Appl. Opt., 49, 1267

Zubko, E., Furusho, R., Kawabata, K., et al. 2011a, J. Quant. Spectr. Rad. Trans., 112,1848

Zubko, E., Muinonen, K., Shkuratov, Y., Videen, G., et al. 2011b, Atti Accad. Pelorit. Pericol. Cl. Sci. Fis. Mat. Nat., 89, C1V89S1P098 